\title{
Design of Damping Base and Dynamic Analysis of Whole Vehicle Transportation based on Filtered White-Noise
}

\author{
GongXue Zhang ${ }^{1, a}$ and Ning Chen ${ }^{2, b,}$ \\ ${ }^{1}$ Shaanxi University of Science \& Technology,Xi'an, China \\ ${ }^{2}$ Shaanxi University of Science \& Technology,Xi'an, China \\ a154854704@qq.com, bchenning4215@qq.com \\ *Ning Chen
}

\begin{abstract}
Keywords:Damping base structure, Dynamics analysis, MATLAB simulation.
Abstract. The design of a eight DOF vibration damping base mechanism and use Creo5.0 with complete assembly process of the base; the structure of the base in the application of the damping spring rod a handicapping combined with tooth tooth fracture energy absorption principle. According to principle of Darren Bell , the model is simplified in the condition of conventional large equipment transportation and the kinematic equation is established, the appropriate vibration model evaluation mechanism is established. By using MATLAB and others software, the time domain model established by filtering and white noise is solved. The accurate simulation analysis is provided for the vertical amplitude, the elevation angle and the inclination angle of the upper end of the base. Finally, by theoretical model building and simulation analysis, the reliability of the base is verified in a certain speed range.
\end{abstract}

\section{Introduction}

With the rapid development of social logistics, a large number of scholars have studied the damping base [1-3]. At present, in the process of transportation of large-scale precision equipment, due to the different incentive of transportation environment, it will produce the reverse road vibration excitation of the packaged goods. The relevant data show that the damage of the goods is that the vibration of the corresponding vibration caused by the corresponding damage to the packaged articles in many ways. With the packaging structure and vibration damping in the development of science, the field is divided into active and passive vibration control of the two methods for large equipment in the field of transportation, rarely specifically design to avoid the trouble for road transport.

Therefore, the design of general vibration absorbing structure is used to reduce the vibration in providing the transportation safety of the corresponding equipment, the means of active vibration reduction are more and more extensive. Under the existing working conditions, a 8 degree of freedom vibration damping base is designed. The speed of the non-uniformity in traffic conditions by simulating the vehicle caused by packaging are vulnerable to a variety of dynamics, which are part of the device design, through the device to realize vibration and fully protected the integrity of shipping goods has become a key in the mechanical device.

\section{Structure design of damping base}

According to the actual transportation conditions, we can distinguish the highway transportation condition into three parts: instrument and equipment, damping base and road excitation. In the kinematic model, the surface excitation can be regarded as the vibration source, the damping base is a damping device, the equipment is the corresponding vibration source. The specific working flow of the vibration damping base is shown in figure 1, which specific conditions. The process of transport vehicles in the road irregularity or vehicle acceleration can generate the corresponding incentive vehicles in the form of transport in the process of transportation equipment. equipment will be shake, the base is connected to the transport packaging through a connecting plate was a corner the end piece, 
the vibration on the base cover through even the keyboard transfer, vibration achieve vibration reduction through two ways. The vibration impact lower by elastic damping and spring damper. The other is that vibration absorption energy when the vibration shock is large. The specific process of tooth fracture energy absorption when the pressure lever is in the amount of time limit, The vibration to the limit plate, the limit plate through the tooth column and vibration damping seat angle of impact energy absorption. After vibration reduction by these two vibration reduction methods, it will be transmitted to the base iron base to carry out the final energy absorption and vibration reduction.

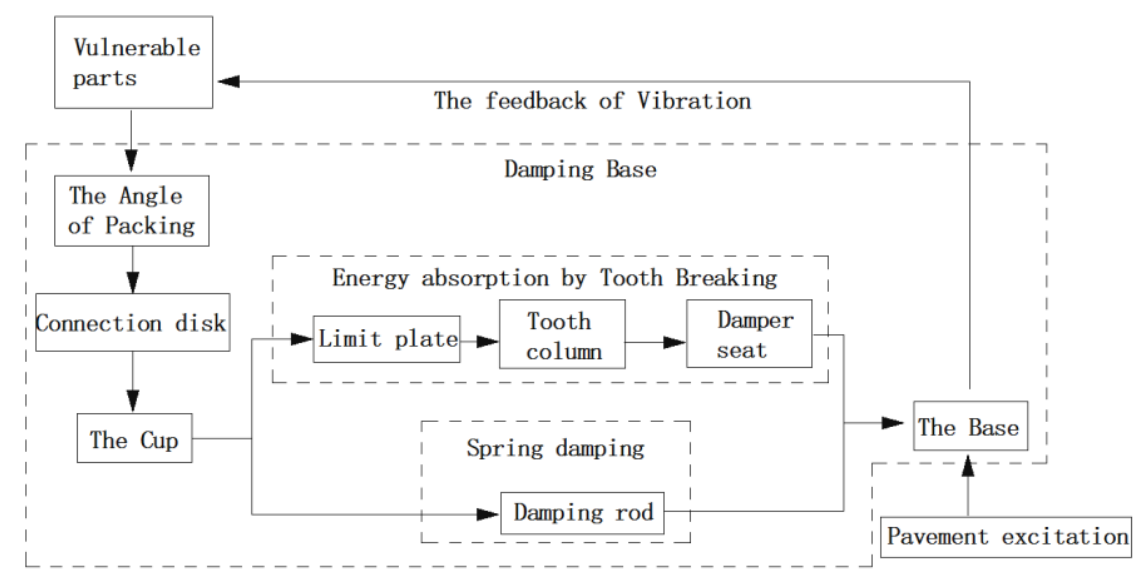

Fig. 1. Specific working flow chart of vibration damping base.

The flexible vibration of machine base, It reduced vibration for the machine in the process of the vibration damping effect by the rigidity and damping vibration damping rod device four angle with the equal distribution of the collision fault vibration of upper middle tooth column. The utility model has the advantages of simple principle, simple structure, low environmental requirement and good vibration damping effect. The structure of the damping base is shown in Figure 2.

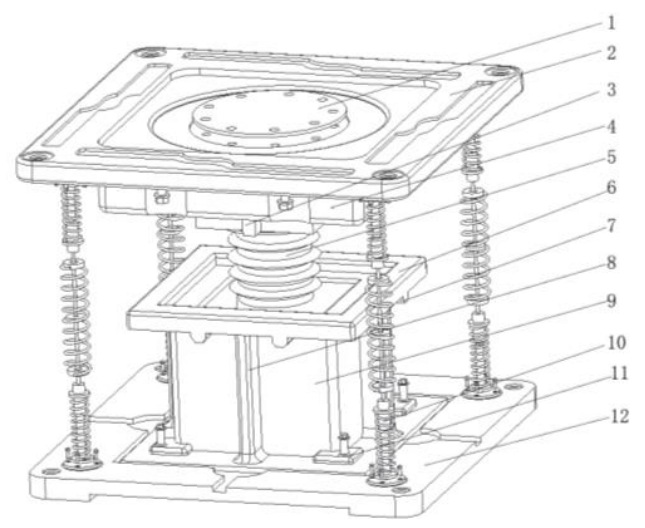

Fig. 2.1 (connection disk), 2 (end cover), 3 (strong stiffener I), 4 (limit disk), 5 (tooth column), 6 (chuck), 7 (stiffener board II), 8 (base), 9 (tooth cylinder), 10 (pressure bar), 11 (fixed hinge), 12 (base).

Sketch of structure of vibration damping base.

\section{Dynamic model of mechanism}

The damping base is composed of a plurality of elastic rod combined tooth column and cast iron cover components such as the center, because the material and a spring bar structure and other factors, so the damping base can not be regarded as a linear system, nonlinear system with multiple degree of freedom, the coupling between the various parts of the whole research base has a composite type. According to related research, when the side transport vehicles in the process of acceleration is less than $0.4 \mathrm{~g}$, the base can be regarded as the same car linear system. In order to simplify the model,We 
make relevant assumptions: (1) The transportation environment of the uneven coefficient is constant and only considering the experimental pavement conditions; (2) Transport vehicles that linear motion movement speed is constant that excluding non linear variable motion movement; (3) The base system for linear systems that is the rod and the tooth column system damping force increases linearly with displacement and velocity; (4) No considering the vibration transfer process of the torque and the corresponding; (5) No considering the fluid base generated in the movement of force. The related parameters are obtained through the above hypothesis and the principle of conservation of energy. In the process of transportation, large equipment movement is divided into upper and lower pitch and skewed three degrees of freedom and the impact damper base four end angle four connecting rod is spring four degrees of freedom and the tooth column system for a total of eight degrees of freedom, the relevant agencies for simplified model. The figure 3 is the kinematics model of the base.

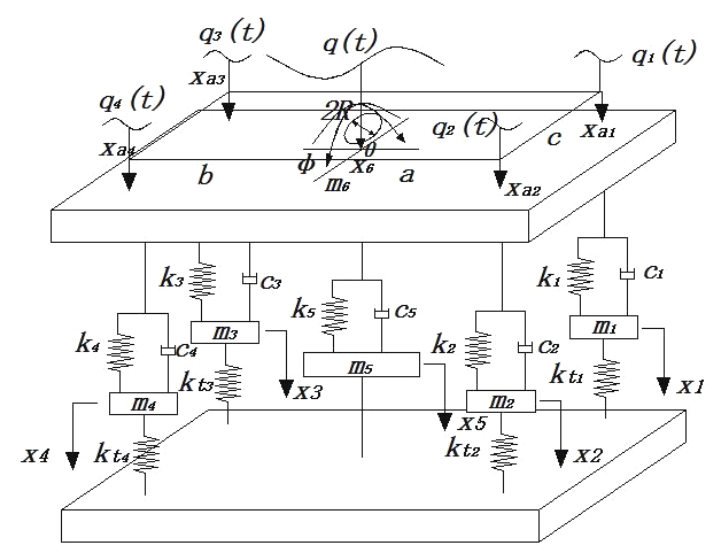

Fig. 3 . Sketch of motion relation between parts on shafts and shafts.

The figure Including:

$X_{1}-X_{4}$ are four spring mass rod vertical displacement in the base;

$x_{5}$ is the vertical displacement of the lumped energy of the tooth column energy absorbing device;

$x_{6}$ is the vertical displacement of the base end cover;

$\theta$ is the base pitch angle displacement;

$\Phi$ is Inclination angle displacement of pedestal;

$k_{l}-k_{4}$ is the base four spring rod two grade equivalent spring stiffness;

$k_{5}$ is the impact stiffness of the tooth column energy absorbing device;

$k_{t l}-k_{t t}$ is a four base elastic rod equivalent spring stiffness;

$c_{1}-c_{1}$ is the base four equivalent damping rod spring;

$c_{5}$ is the equivalent damping of the gear column energy absorbing device;

$a$ and $b$ are connected to both ends of elastic rod from disk.

The displacement pressure rod connecting position in the vertical direction and the end cover showed by (1).According to Darren Bell's principle, the differential equations of vibration in the vertical direction of five non spring loads are obtained and it's showed by (2):

$$
\left\{\begin{array}{l}
x_{\mathrm{a} 1}=x_{6}-a \theta+\frac{1}{2} c \varphi \\
x_{\mathrm{a} 2}=x_{6}-a \theta-\frac{1}{2} c \varphi \\
x_{\mathrm{a} 3}=x_{6}+b \theta+\frac{1}{2} c \varphi \\
x_{\mathrm{a} 4}=x_{6}+b \theta-\frac{1}{2} c \varphi
\end{array}\right.
$$

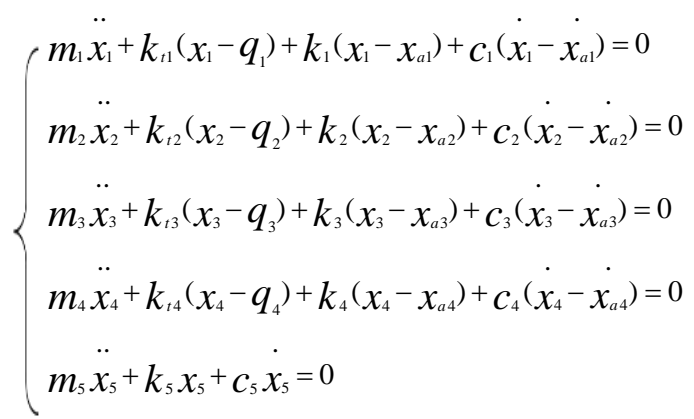


The vertical vibration differential equation of the base end cover is: $m_{6} \ddot{x_{6}}+k_{1}\left(x_{a 1}-x_{1}\right)+k_{2}\left(x_{a 2}-x_{2}\right)+k_{3}\left(x_{a 3}-x_{3}\right)+k_{4}\left(x_{a 4}-x_{4}\right)+k_{5}\left(x_{a 5}-x_{5}\right)+c_{1}\left(\dot{x_{a 1}}-\dot{x_{1}}\right)+c_{2}\left(\dot{x_{a 2}}-\dot{x_{2}}\right)+c_{3}\left(\dot{x_{a 3}}-\dot{x_{3}}\right)+c_{4}\left(\dot{x_{a 4}}-\dot{x}_{4}\right)+c_{5}\left(0-\dot{x_{5}}\right)=0$

The differential equation of pitching vibration of the base end cover is:

$I_{p} \ddot{\theta}+\left[k_{3}\left(x_{a 3}-x_{3}\right)+c_{3}\left(\dot{x_{a 3}}-\dot{x}_{3}\right)+k_{4}\left(x_{a 4}-x_{4}\right)+c_{4}\left(\dot{x_{a 4}}-\dot{x_{4}}\right)\right] b-\left[k_{1}\left(x_{a 1}-x_{1}\right)+c_{1}\left(\dot{x}_{a 1}-\dot{x}_{1}\right)+k_{2}\left(x_{a 2}-x_{2}\right)+c_{2}\left(\dot{x_{a 2}}-\dot{x_{2}}\right)\right] a=0$

The roll vibration differential equation of the base end cover is:

$I_{r} \ddot{\varphi}+\left[k_{1}\left(x_{a 1}-x_{1}\right)+c_{1}\left(\dot{x_{a 1}}-\dot{x_{1}}\right)+k_{3}\left(x_{a 3}-x_{3}\right)+c_{3}\left(\dot{x_{a 3}}-\dot{x_{3}}\right)-k_{2}\left(x_{a 2}-x_{2}\right)+c_{2}\left(\dot{x_{a 2}}-\dot{x_{2}}\right)-k_{4}\left(x_{a 4}-x_{4}\right)-c_{4}\left(\dot{x_{a 4}}-\dot{x_{4}}\right)\right] \frac{1}{2} c=0$

When a section of road condition is known, all the vibration of one end of the base is stable and the amplitude is not more than $0.02 \mathrm{M}$. The safety limit frequency range of the relevant instrument and equipment meets the requirements.

According to a truck at a certain speed traction driving,the corresponding vibration white noise input and went in front of the formula simulation model was established and the link gain by the construction of the system model in MATLAB, will be affected by the external excitation of $q(t)$ in the process of the movement of the trajectory.It can be the external incentive through related transfer functions scattered to the four corner, mainly connected on the base of vertical displacement, the base pitch angle and the base side angle gain link specific simulation process as showing figure 4 .

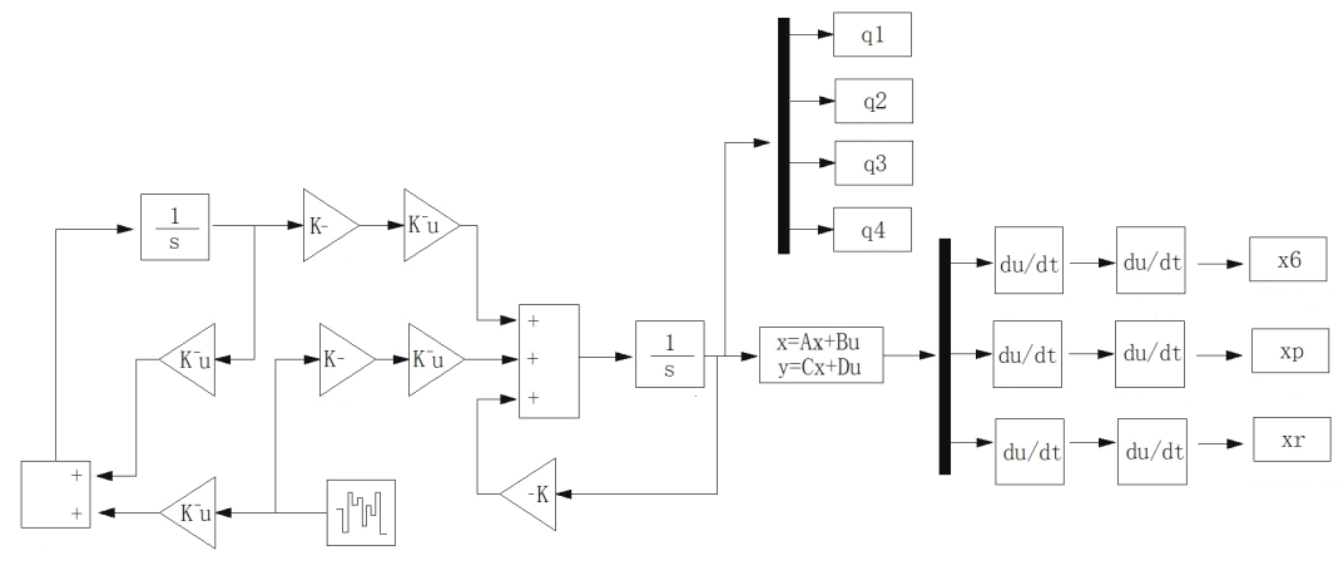

Fig. 4 . Simulation model

By building the simulation model, in the selection of truck speed change in the conventional $40 \sim 50 \mathrm{~km} / \mathrm{h}$, we knew that the ending limit amplitude control under $0.02 \mathrm{M}$, the amplitude meet the safe transport of large equipment. The base for the ultimate displacement of the damping effect in the vertical direction meet the requirements by the ending ${ }^{[4]}$ It shows that the pitch angle range is 0.004 0.0085rad through access to relevant information but also meet the requirements of safe transportation of large equipment pitch angle; changing the scope of the side angle is $0.0042 \sim 0.0085 \mathrm{rad}$ through access to relevant information and meet the safety requirements of large equipment transport side angle by the ending. In conclusion, as the base is designed as a square damping mechanism, it is also found that the range of pitch angle and side angle varies little by contrast.By compared with other literature ${ }^{[5]}$, we are showed the reliability of the simulation.

\section{Ending}

(1) The design of a eight DOF vibration damping base mechanism is built and use Creo5.0 with complete assembly of the base; Designing that a spring damping rod and column handicapping combined tooth tooth breaking energy absorbing principle application. (2) According to Darren Bell's principle, the model is simplified and the kinematic equations are established and the appropriate 
vibration model evaluation mechanism is established. By using MATLAB and other software, the time domain model established by filtering and white noise is solved and the reliability of the base is explained. (3)Through theoretical model and simulation demonstration, the reliability of vibration reduction of this base is confirmed which provides theoretical support for the subsequent application in actual productions and foundations.

\section{References}

[1] L. De-Li, The influence of the structure of the base structure on the vibration characteristics of the hull, Ship mechanics, vol.05, pp. 557-566, 2012.

[2] Z. Shu-Lei, Research on damping performance of base damping, Material development and application, vol.02, pp. 27-31, 2014.

[3] Y. Ji-Kuan, Vibration reduction design of mechanical devices with non rigid base, Journal of Shanghai Jiao Tong University, vol.03, pp. 83-94, 1964.

[4] Z. Gong-Xue, Eleven degree of freedom vehicle dynamics model and comfort simulation, Mechanical design and manufacture, vol. 01, pp. 43-46, 2017.

[5] C. Hong-Yue, Nonlinear vibration analysis of 7 degree of freedom shearer under working condition, mechanical strength, vol. 01, pp. 1-6, 2017. 